\title{
Lidil
}

Revue de linguistique et de didactique des langues

$64 \mid 2021$

Le passif dans la langue parlée

\section{Étude microsyntaxique et macrosyntaxique des verbes de parole à l'oral employés au passif}

Microsyntactic and Macrosyntactic Study of Speech Verbs in Passive Uses

\section{Badreddine Hamma}

\section{(2) OpenEdition}

12 Journals

Édition électronique

URL : https://journals.openedition.org/lidil/9468

DOI : $10.4000 /$ lidil. 9468

ISSN : 1960-6052

Éditeur

UGA Éditions/Université Grenoble Alpes

Édition imprimée

ISBN : 978-2-37747-315-1

ISSN : $1146-6480$

Référence électronique

Badreddine Hamma, «Étude microsyntaxique et macrosyntaxique des verbes de parole à l'oral employés au passif », Lidil [En ligne], 64 | 2021, mis en ligne le 01 novembre 2021, consulté le 25 novembre 2021. URL : http://journals.openedition.org/lidil/9468 ; DOI : https://doi.org/10.4000/lidil. 9468

Ce document a été généré automatiquement le 25 novembre 2021.

(c) Lidil 


\title{
Étude microsyntaxique et macrosyntaxique des verbes de parole à l'oral employés au passif
}

Microsyntactic and Macrosyntactic Study of Speech Verbs in Passive Uses

\author{
Badreddine Hamma
}

\section{Introduction}

Dans ce numéro sur les apports de l'oralité et des corpus oraux à l'étude du passif, on ne peut pas éviter la question du statut grammatical global de ce tour et de son étendue dans un discours non contrôlé. En effet, le passif n'est pas toujours un segment phrastique supérieur et autonome. Ce point avait déjà été évoqué dans des études assez récentes (cf. Hamma, 2015, 2017, 2020a et 2020b ; Hamma et coll., 2017) et mérite d'être approfondi ici. Ainsi, le passif a toujours été considéré d'une manière plus ou moins explicite comme consubstantiel et coextensif au déploiement de la phrase dans les descriptions existantes (entre autres, Dubois, 1967 ; Brahim, 1996 ; Gaatone, 1998). Dans les grammaires de référence, on parle aussi presque invariablement de «phrase (à la voix) passive». Toutefois, confrontée aux données orales, l'étiquette "phrase» pourrait paraitre quelque peu déplacée et inopérante, surtout si l'on part du principe largement répandu qu'une phrase est l'unité supérieure dotée d'une intégrité sémantique et formelle isomorphe et qui serait, de ce fait, autonome et autosuffisante : une idée par phrase (cf. Bloomfield, 1933-1970 ; Le Goffic, 1993, 2016 ; Riegel et coll., 2009-1994). Il existe en effet de nombreux phénomènes linguistiques qui ne peuvent pas être pris en charge par la syntaxe de la phrase, car échappant à la rection verbale (cf. Blanche-Benveniste \& Jeanjean, 1987 ; Berrendonner, 1990, 2011 ; Dik, 1989 ; Adam, 2005 ; Rossi-Gensane, 2010 ; Haselow, 2016 ; voir aussi Béguelin, 2002 ; Sabio, 2006 ; Avanzi, 2007 ; Benzitoun, 2010). Il a été alors proposé divers autres types de découpages du discours, comme "paragraphe oral", "période", "hyperphrase", " regroupement", «clause ", etc. On retrouve aussi des appellations moins marquées, 
comme "séquence» ou «segment ", visant à décrire des portions de discours non couvertes ou ne coïncidant pas nécessairement avec la phrase : leurs portées et leurs frontières sont définies à l'aide de critères faisant appel entre autres à la pragmatique, au sens et aux isotopies lexicales (cf. Berrendonner, 2003; Adam, 2005), ainsi qu'aux considérations suprasegmentales (Morel \& Danon-Boileau, 1998). Notre démonstration, ici, portera sur les verbes de parole construits au "passif périphrastique ${ }^{1}$ " à l'oral. Notons toutefois qu'il ne sera pas question non plus de se passer de la notion de "phrase", qui reste opérante sur le plan microsyntaxique: chaque passif périphrastique constitue théoriquement une phrase, sans nécessairement être autonome sur le plan discursif.

\section{Choix méthodologiques et constitution du corpus}

2 Notre approche s'inscrit dans une perspective distributionnelle appliquée aux passifs périphrastiques observables dans de vraies productions verbales. Nous y considérons de près la relation du verbe avec les divers "rôles actanciels " ou "circonstants " en jeu, en termes de sélection sémantique et de sous-catégorisation syntaxique. Quand ces rapports n'apparaissent pas en surface, nous pouvons généralement les restituer à travers certaines opérations linguistiques, comme la paraphrase, les enchainements possibles, le remplacement ou aussi l'insertion. Ce type d'information fait partie de la compétence linguistique des sujets parlants et implique la mémoire collective et les savoirs partagés dans une communauté donnée. Pour désigner ces rapports, nous utiliserons les termes «formes profondes » vs «de surface» ou "actants latents » (traduisant "actant slots») tels qu'utilisés dans la théorie Sens-Texte (cf. Mel'čuk, 2004). Ainsi, le verbe parler, dans un énoncé comme il a parlé, peut être mis en relation, dans un contexte donné, avec une projection actancielle assez stable: "qqn qui a parlé ", " qqn à qui l'on a parlé ", " qqch qui a été dit ", mais aussi, selon les cas, avec un mode ou un support de "parole " (cf. téléphone, courrier...), ou une manière de "parler/dire» (fort, avec prudence, discrètement, en chuchotant, avec fermeté, poétiquement...), ainsi que d'autres informations qui peuvent paraitre moins centrales pour le procès parler (temps, lieu, etc.). Ces considérations se fondent exclusivement sur des traces linguistiques dans les échanges réels et prennent en considération le sens en contexte. Dans des échanges in situ, il est rare que toutes ces informations soient présentes en surface de manière explicite pour différentes raisons pragmatiques (pertinence, économie, qualité...). Pour ce qui est de la description de l'oral, nous reprendrons surtout les outils et dispositifs de description proposés par les modèle aixois (cf. Blanche-Benveniste et coll., 1984 ; Blanche-Benveniste, 2010) et fribourgeois (cf. Groupe de Fribourg, 2012), avec parfois quelques ajustements.

3 Par souci de transparence, nous faisons le choix, ici, de nous arrêter sur toutes les décisions significatives intervenant dans la sélection des données, aussi bien au niveau des requêtes et de l'extraction des formes ciblées qu'au niveau des opérations de tri auxquelles nous avons procédé. Cela permettra à la fois de suivre de très près la démarche adoptée et d'éclairer davantage les conclusions de ce travail. 


\section{1. À la recherche des verbes de parole au passif dans les ESLO}

Notre corpus s'appuie sur une sélection initiale de 4887521 mots des ESLO $1 \& 2^{2}$. Et, comme outil d'extraction, notre choix s'est arrêté sur TXM ${ }^{3}$. Afin d'avoir une quantité de données raisonnable pour le présent travail, nous avons opté pour une restriction typologique et morphosyntaxique ciblant les verbes dits de parole employés au passif périphrastique. Ces verbes sont très courants et se prêtent parfaitement à des dispositions au passif. En effet, un grand nombre des verbes de parole (désormais VP) sont transitifs (le " dire » s'appliquant généralement à une " production verbale »); or, la transitivité, qu'elle soit fournie en surface ou impliquée comme "position latente " des énoncés, est particulièrement significative pour les constructions étudiées (Gaatone, 1998). Pour déterminer la liste des VP à rechercher dans TXM, nous nous sommes appuyé sur les tables 6 et 9 de Gross (1975), ainsi que sur certaines études plus récentes sur les VP, en particulier, Mathieu-Colas (2006), Lamiroy et Charolles (2008), ou aussi Anscombre (2015). La liste définitive retenue s'élève à 79 verbes. Elle a été actualisée, adaptée et stabilisée par nos soins pour une application à un français oral non lu, celui des ESLO. Ont été exclus, par exemple, les verbes qui décrivent surtout une « manière de parler », en particulier, ceux qu'Anscombre (2015, p. 108) appelle « verbes phonatoires ", qui sont surtout intransitifs, ce qui ne conviendrait pas à notre étude (cf. bégayer, zozoter, bredouiller, vociférer, balbutier, baragouiner, bafouiller, marmonner, cafouiller, etc.) et qui allaient de toute façon être évincés de par leurs propriétés distributionnelles. Ne seront pas non plus associés à la recherche les verbes ne s'appliquant pas à un objet précis (cf. délirer, divaguer, rouspéter, fulminer, etc.). Le pivot de nos requêtes <[frlemma= "VP (dire|parler...)" \& frpos="VER:pper"]> (cf. infra) était supposé cibler les périphrases verbales constituées de l'auxiliaire être dans ses différentes actualisations, suivi immédiatement d'un verbe au participe passé (désormais Vppé), appartenant à notre liste de 79 verbes de parole.

5 Pour établir la chaine précise de notre requête, il fallait définir aussi d'autres critères additionnels de sélection visant à écarter les cas ne se prêtant pas à une analyse en termes de diathèse passive de type périphrastique. Parmi ces cas indésirables et outre les verbes attributifs, on pourrait citer, en particulier, les nombreux verbes pronominaux à sens passif ou non, qui, en se construisant avec l'auxiliaire être, prennent une forme proche des passifs périphrastiques, qu'ils soient réfléchis, réciproques ou moyens, du type se parler, s'expliquer, se dire, s'exprimer, etc. Leur exclusion est exprimée dans la syntaxe de la requête par le point d'exclamation «! » affectant la liste nominative que TXM symbolise par l'étiquette word (renvoyant aux clitiques indésirables $m e$, se et te) et correspondant en l'occurrence à la position préverbale canonique P2 (position des proclitiques objet dans l'approche pronominale ; cf. Blanche-Benveniste et coll., 1984), avec un empan adapté, allant de zéro à trois mots $\langle\{0,3\}>$. Et, pour les proclitiques homonymiques nous et vous, nous nous sommes résolu à les enlever manuellement en aval. Voici la forme définitive de la requête saisie dans TXM (avec nos 79 verbes de parole) :

$<[$ word!="s'|m'|se|me|t'|te|s""]\{0,3\}[frlemma="être"][frlemma="accuser|affirmer| alerter|alléguer|annoncer|attester|avertir|aviser|avouer|balancer|calomnier| certifier| citer|commander|confesser|confier|confirmer|conseiller|conter|contester| critiquer| débattre|déclarer|déconseiller|demander|démentir|dénigrer|désavouer| désigner| dévoiler|dicter|dire|discuter|divulguer|énoncer|évoquer|exiger|expliquer| exposer| exprimer|formuler|gronder|implorer|incriminer|indiquer|informer| injurier|insulter| interroger|invoquer|maudire|menacer|mentionner|nommer| 
ordonner|prétendre| prétexter|prévenir|proférer|promettre|prononcer|proposer| questionner|raconter| rappeler|réclamer|recommander|redire|reformuler|refuser| réfuter|relater|répéter| répondre|révéler|revendiquer|signaler|vendre| parler|"\&frpos="VER:pper"] >4 .

6 Du point de vue sémantique, les VP retenus rentrent tous dans des projections actancielles sous-catégorisant trois rôles sémantiques possibles, représentés par trois actants bien distincts (désormais ACT) s'actualisant sous forme de dépendants spécifiques qui varient en fonction du type de disposition (active ou passive) : le NO (le sujet), le N1 ( $1^{\text {er }}$ argument interne du verbe) et le N2 ( $2^{\text {nd }}$ argument interne du verbe) :

- $($ ACT1) « celui qui dit quelque chose » (une « source » ou un « agent »);

- $(\mathrm{ACT} 2)$ « ce qui est dit » (« une production verbale») ;

- $(\mathrm{ACT} 3)$ « celui à qui l'on dit quelque chose » (« un destinataire »).

7 Dans notre étude, c'est le concours des interfaces morphosyntaxique, phonologique et sémantique, avec le renfort de la pragmatique, qui permet d'avoir une saisie plus complète des usages des VP au passif dans le discours. Le recours à une telle vision globale s'explique par l'absence d'isomorphisme entre ces dimensions : ainsi, le rôle sémantique correspondant à "l'agent " pourrait s'actualiser à la fois comme complément d'agent (par N2) et comme sujet (N0) et les considérations pragmatiques et discursives peuvent expliquer la présence ou non de certains des actants en jeu ou aussi l'orientation de leur force illocutoire. Enfin, la structure prosodique concourt à une meilleure description des liens macrosynatxiques.

\subsection{Résultats de nos requêtes et bilan des opérations de tri}

8 Le premier résultat obtenu était de 517 occurrences. Et, comme nous pouvions nous y attendre, nous nous sommes aperçu, suite à un premier examen de ces résultats, qu'il y avait toujours des cas indésirables ayant échappé aux filtres de notre requête et qu'il était donc nécessaire d'éliminer. Nous avons en particulier relevé de nombreux cas de passifs pronominaux de VP en $" s^{\prime} / m^{\prime} / t^{\prime} »$ ( 120 occurrences), ce qui constitue visiblement un défaut d'annotation dans TXM au niveau des élisions. Un tri croissant par ordre alphabétique de la colonne représentant le pivot a permis de les repérer facilement. Sur les 397 occurrences restantes, il y avait de nombreux autres cas indésirables qu'il fallait aussi traiter. La polysémie de certains verbes, par exemple, nous a donné du fil à retordre dans notre travail de fouille en nous obligeant à les voir au cas par cas. En effet, de nombreux verbes pouvaient s'appliquer aussi bien à une personne qu'à un objet non verbal (cf. confier, nommer qqn/qqch; exposer, proposer qqch; qqn qui est confirmé/certifié/avoué; etc.). Il en va de même de certains autres verbes où ce qui faisait office d'ACT2 s'avérait hybride. Ainsi, certains emplois du verbe refuser, signifiant "dire non ", s'appliquaient aussi à des contenus non verbaux (un cadeau, une promotion, la drogue, etc.), ou portaient sur une tierce production verbale : dire non à une offre, à un conseil, à une demande, à une requête, etc.) - cas aussi de verbes, comme réfuter, démentir, confirmer, confier, etc. À tout cela s'ajoutaient quelques cas d'erreurs de transcription que nous avons aussi éliminés manuellement (la locution verbale de déplacement avec le pronom personnel il « avoir été $+V$ inf » dans Il a été demander qqch à qqn ayant été transcrite à deux reprises *il a été demandé qqch à qqn). 
9 Notons, par ailleurs, que certains verbes se sont imposés à nous comme des VP par association à un verbe comme dire dans le cadre d'une reformulation, d'un entassement paradigmatique (cf. Blanche-Benveniste et coll., 1984) ou d'une recherche du mot juste (cf. répandre, atténuer, poser, broder, détailler, etc.). Nous avons fait le choix de ne pas les intégrer dans notre requête étant donné que leur sens comme VP était accidentel. Nous avons, en revanche, tenu compte de ces verbes dans l'analyse. Les verbes les plus fréquents étaient dans l'ordre : dire (56 fois), demander ( 29 fois), décider (26 fois), parler (20 fois), évoquer (11 fois), discuter (10 fois), suivis de expliquer, prononcer, déclarer, proposer, répondre, raconter, reprendre, redire, etc., avec des fréquences moins importantes. En dernier ressort et après avoir écarté à la main tous les cas indésirables, il n'est resté que 255 passifs avec des VP entrant dans le schéma retenu, que nous considérons comme base ici pour l'étude entreprise.

\section{Pour une description micro et macrosyntaxique des passifs périphrastiques des verbes de parole}

Bien que les verbes de parole employés au passif retenus répondent tous globalement à une distribution actancielle tripartite, il faudrait préciser que leur emploi dans le discours prend des formes très variées. Cela va d'une réalisation maximale avec les trois arguments de surface (N0 VP $\mathrm{PASSIF}_{\mathrm{a}}$ N1 par N2), comme en (1), à une réalisation partielle avec divers cas d'économie, comme l'illustrent les exemples (2-5) ci-dessous. Les arguments N1 et 2 peuvent ainsi être absents en surface tout en restant parfaitement accessibles par inférence ${ }^{5}$. L'absence intermittente de certains de ces arguments peut s'expliquer par différentes raisons : l'information peut être évidente, peut avoir été dite ou partagée ou peut être établie à partir du contexte immédiat linguistique et/ou situationnel. Ainsi, on peut aisément inférer de l'exemple (2) que ce sont des acteurs de l'Éducation nationale qui auraient pu faire la remarque sur la qualité de l'orthographe du locuteur (cf. inspecteurs, directeur de l'établissement, collègues, etc.). Cette absence peut aussi relever d'une stratégie discursive visant à taire délibérément cette information par calcul ou par discrétion (cf. notion d' " occultation » chez, entre autres, Brahim, 1996) :

(1)

ça ça m'a été raconté par euh ma grand-mère qui était oui qui était totalement illettrée

(2)

je vous dis ce qui m’a été dit quand je suis rentrée à l'éducation nationale

(3)

ce n'est pas tellement ce qui peut être dit par tel ou tel maitre

(4)

le thème déjà et la [oui] façon dont c'est filmé dont c'est raconté

(5)

comme là tout est expliqué

11 L'examen des données montre que les VP ne constituent pas non plus une classe homogène sur le plan sémantique, d'où l'intérêt de les considérer distinctement sur ce plan. C'est, en effet, ce type de rapports qui dicte globalement la sélection des noms en jeu (N0, N1 et N2). 


\subsection{Sous-classes des verbes de parole employés}

12 Pris dans l'absolu, ce sont surtout les VP les moins marqués qui permettent une réalisation potentiellement complète en surface, du type: dire, exprimer, informer, annoncer, indiquer, etc.; le sens du verbe informe seulement sur la transmission de la parole et éventuellement sur le canal utilisé et non sur l'objet communiqué (cf. est-ce un conseil, une question, une mise en garde, etc. ?). Les autres VP, qui semblent plus spécifiques (cf. Lamiroy \& Charolles, 2008), peuvent se rapporter, quant à eux, à des cas où il est fait allusion à l'ACT2 (la parole dite) dans le sens lexical du verbe lui-même. C'est le cas des verbes réfuter, refuser, accepter, expliquer, conseiller, déconseiller, critiquer, proposer, insulter, interroger, etc., comme ceux de (6-10), qui se laissent tous paraphraser par des tournures renfermant un verbe support et un nom apparenté morphologiquement ou sémantiquement au VP plein, du type «exprimer un refus; dire non/oui ; donner une explication/un conseil ; faire une critique/une proposition ; proférer des insultes/des menaces ; poser une question, etc. ». Le N1 de la disposition passive peut dans ce type de cas ne pas renvoyer directement à la parole dite en surface mais, par exemple, à l'ACT3 (le destinataire), ce qui rend la position N2 à l'actif caduque ou en fait une position non régie par le verbe (avec un détachement gauche ou droit). Ainsi, le VP expliquer peut s'analyser comme la succession d'un verbe support, comme donner, fournir ou livrer et d'un $\mathrm{N}$ « prédicatif » (Gross, 1981), comme (une) explication. Il en va de même de verbes comme discuter et débattre, qui impliquent la présence d'un échange verbal dans les deux sens, pris en charge au moins par deux co-énonciateurs à tour de rôle, ce qui peut être exprimé généralement par un complément d'agent indéterminé à sens collectif ou par un pluriel, ou aussi par un passif « court » (Dubois, 1967, p. 25) et où l'absence de l'agent s'interprète comme un emploi générique :

(6) et l'examen se passe en présence du professeur toujours les élèves sont interrogés par deux deux professeurs dont le professeur qui les a suivis pendant quatre ans

(7)

euh enfin tout ce qui peut être expliqué je pense

(8)

je crois que les programmes doivent doivent pas être discutés mais alors l- le la la classe peut être discutée

(9)

ils veulent pouvoir se diriger eux-mêmes mais éventuellement ils acceptent d'être conseillés voilà

(10)

un autre établissement d'Orléans a demandé plusieurs sections qui lui ont été refusées

De cette dernière classe de VP se dégage une autre sous-classe renvoyant à des noms humains apparaissant dans la position N1 et devant s'interpréter dans leur structure profonde comme faisant référence de manière indirecte à une production verbale adressée à la personne humaine en question (ACT3). On les trouve avec des VP comme prévenir, avertir, menacer, informer, conseiller, déconseiller, aviser, calomnier, accuser, etc. Dans ce type d'emploi, le VP peut sélectionner un complément secondaire en surface, souvent en $\mathrm{de} /$ sur $\mathrm{N}$ ou que-P ou toute autre formulation équivalente (11-13), ayant trait à un « objet de parole » :

(11)

c'est-à-dire que vous on a été averti de votre visite 
(12)

ils ont été aussi prévenus que ils étaient les très bienvenus

(13)

ce qui fait qu'ils sont interrogés sur ces questions traitées

14 Certains VP que l'on rencontre surtout dans le discours rapporté indirect (14-16) renferment une manière de dire les choses : évoquer, atténuer, poser, détailler, prétendre, invoquer, exiger, contester, confier, etc. De tels verbes livrent souvent une idée sur l'attitude énonciative et sur le degré d'adhésion à ce qui est rapporté (cf. Elduayen, 2007 ; Anscombre, 2015), ou témoignent d'un certain effort d'interprétation lors de la reformulation des propos relayés (14-18) :

(14)

ce qui a été dit dans le préambule a été très fortement atténué dans les textes de la loi

(15)

bon ça a été évoqué là dans le dans l'Express

(16)

elle m'a dit non on est tous au clair donc euh voilà les choses sont posées sont dites tout de y a pas de cachoteries

(17)

sur l'ouvrage tout étant détaillé hm sur l'ouvrage

(18)

ça a été un petit peu brodé par les gens

15 Un autre type de VP a pour source un N non humain (panneau, livre, lettre, document, etc.), mais qui est aussi relié, dans l'interprétation, à une source humaine (19-22) : on a une personne qui indique, souligne, raconte, décrit, etc., en utilisant un support écrit (ou filmé dans 20). Dans ce type de constructions, un agent humain peut soit supplanter le N2 en question, soit être inséré comme un vrai agent quand sa précision est pertinente sur le plan discursif. D'après les données ESLO, le support du message communiqué peut prendre la forme d'un complément d'agent métonymique en par (21-22) et peut aussi prendre la forme d'un locatif se construisant avec une préposition, comme dans ou sur (cf. 23-24), indiquant la source :

(19)

vous suivez toujours le boulevard et la mairie après est sur votre droite [OK] c'est indiqué [d'accord merci] mais y a au moins un kilomètre à pied

(20)

le thème déjà et la [oui] façon dont c'est filmé dont c'est raconté

(21)

et selon des procédures qui lui sont également indiquées euh par les textes c'est-à-dire qu'on ne peut pas nommer n'importe qui à n'importe quel emploi pour un emploi euh comme le mien par exemple il faut posséder euh des qualifications euh universitaires

(22)

ensuite vous arriverez sur la place de la cathédrale et ça sera indiqué ensuite par les panneaux [OK bah merci beaucoup merci au revoir]

On peut relever par ailleurs quelques usages particuliers de VP entrant dans des constructions impersonnelles. Ils renvoient généralement aux tours dits « occultifs » (cf. Brahim, 1996), où la mention de l'agent, effectivement, ne semble pas au cœur de l'échange bien que restant accessible dans le contexte. Ce sont des cas où la source de la parole coïncide avec une autorité, un cadre plus restreint, une instance administrative (23-25) ou alors avec une source plus large (une communauté donnée, le sens commun, la mémoire discursive, etc.) : 
(23)

sur la fiche technique il est dit comédie moi je veux bien le croire

(24)

dans ce grand préambule il était dit en particulier qu'il ne s'agissait nullement de vouloir faire rentrer l'enseignement catholique dans l'éducation nationale

(25)

un jour je crois qu'ils ont demandé un exemple des cendriers dans les vestiaires de femmes il a été répondu que les les femmes [oui] n'avaient pas besoin que normalement les femmes ne fumaient pas et que c'était pas esthétique de mettre des cendriers

Enfin, il conviendrait de signaler aussi la présence d'un emploi très particulier de certains VP, surtout avec dire (26-29), renvoyant à un usage passif attributif lexicalisé que l'on peut paraphraser par "réputé $\mathrm{x}$ » ou "connu sous le nom $\mathrm{x}$ ». Ces emplois utilisent généralement un temps non accompli (ici le présent) et ont une valeur non occurrentielle (exprimant un «passif d'état ») :

(26)

y a des congrès euh locaux hm qui sont dits parfois de bonne qualité on fait venir des intervenants de de de France entière

(27)

il y a des régions de France qui sont dites des régions euh très chrétiennes

(28)

y a euh tout de même des des gens qui sont dits euh tenir comme on dit le dessus du pavé ça d'abord ça ne dure pas

(29)

mais quels sont ces gens qui sont dits cultivés? [ah ben c'est l'élite de la ville comme on dit l'élite culturelle justement vous comprenez]

En somme, les différents VP relevés dans notre corpus s’inscrivent tous dans le schéma défini plus haut impliquant l'auxiliaire être et un participe passé. Selon les arguments sélectionnés, leurs classes et leur degré de lexicalisation, le VP passif peut être situé sur une échelle de processivité impliquant directement ou indirectement un agent humain volontaire (ACT1), s'appliquant à une production verbale comme objet (ACT2) et renvoyant dans certains cas à une information oblique que l'on peut restituer indirectement à partir des distributions observables. Par ailleurs, dans tous ces cas de figure, il y a toujours un ACT3 (un destinataire), même s'il constitue la plupart du temps une information sous-entendue que le contexte permet aisément d'établir. Notons, enfin, que, si l'ACT1 et l'ACT3 peuvent ne pas avoir d'actualisation en surface dans une disposition au passif, l'ACT2, quant à lui, est indispensable dans les constructions personnelles, dans la mesure où il fait partie de la réalisation minimale des passifs périphrastiques en français.

\subsection{Sur le statut microsyntaxique et macrosyntaxique du passif périphrastique}

Si nous utilisons "segment passif» dans cette étude pour parler des passifs périphrastiques de notre corpus, c'est parce que ce tour ne correspond pas nécessairement à une phrase autonome et autosuffisante. Il peut, en effet, relever d'une séquence hypotaxique régie par un composant phrastique plus important (cf. je suis sûre qu'ils entendent pas cequi est raconté) ou correspondre, à l'inverse, à des dépendants associés de type macrosyntaxique avec des liens pragmatiques et sémantiques, échappant à la syntaxe de la phrase et que l'on décrit parfois comme 
des cas de parataxe (cf. bah il y a le l'arabe il est parlé à Orléans). Notons cependant que l'étiquette "segment passif» retenue ici faute de mieux, bien que recoupant partiellement les notions "regroupement illocutoire" (dans le modèle aixois) et " clause » (dans le modèle fribourgeois), ne s'y réduit pas; les passifs relevés n'étant pas toujours des segments syntaxiques autonomes comme le sont à priori ces dernières, ni nécessairement la partie centrale de l'échange. Ils peuvent relever d'une simple séquence régie par un nœud verbal, nominal ou adjectival (voir infra) et non nécessairement d'un segment indépendant. De fait, il sera assez commode de décrire ces rapports en termes de "noyau », de "pré-noyau » et de "post-noyau », en suivant en cela le modèle aixois dans la description de l'oral (cf. Blanche-Benveniste, 2010), mais sans perdre de vue les aspects microsyntaxiques et hypotaxiques en jeu.

\subsubsection{Le passif périphrastique dans les relations hypotaxiques}

Sur les 255 segments passifs retenus, il y a 128 occurrences, soit 50,19\% de l'ensemble, qui sont des éléments hypotaxiques. Ces dernières ne constituent pas des phrases passives à part entière, mais de simples fragments phrastiques. Ainsi, les exemples (30-32) relèvent de compléments de nom, qui sont dans 67 cas une relative en qui, où ou dont et se rattachent, en l'occurrence, à un nom souvent non saturé (des $\mathrm{N}$ collectifs indéterminés ou des $\mathrm{N}$ indéfinis) et la relative passive vient justement le développer, l'expliquer ou en restreindre le sens :

(30)

et euh dans le cadre du de notre projet là donc euh on s'intéresse à la langue et on s'intéresse aussi aux différentes langues qui sont parlées à Orléans $[\mathrm{hm} \mathrm{hm]} \mathrm{et} \mathrm{euh} \mathrm{par}$ rapport à ça est-ce que tu as déjà fait attention euh à d'autres langues qui seraient parlées dans Orléans quand tu te promènes ou quand?

(31)

le thème déjà et la [oui] façon dont c'est filmé dont c'est raconté

(32)

en France on prétend que c'est à Tours surtout dans l'Indre-et-Loire et peut-être l'Orléanais [hm hm] où le français est parlé le plus correctement avec le moins d'accent

Parmi ces constructions, on a relevé 23 relatives passives entrant dans un « dispositif auxiliaire de la détermination nominale ", selon les procédés aixois, avec un N indéfini, en (33), sans doute pour éviter d'avoir des $\mathrm{N}$ indéfinis comme sujet, ce qui aurait été acceptable à l'écrit (cf. des choses énormes étaient dites...), ou aussi un dispositif de clivage, en (34):

(33)

$y$ avait des choses énormes [hm] qui étaient dites jusqu'à un sous-marin dans la Loire des trucs incroyables [hm hm]

(34)

euh là c'est le baccalauréat baccalauréat qui est exigé

L'intervention d'une relative passive peut s'étendre aussi à 20 autres cas en lien avec le pronom démonstratif, dit neutre, ce qui constitue une tête de syntagme non saturée, formant avec la relative passive, obligatoire ici, un syntagme nominal en (35-37). Ces emplois sont reconnaissables à la possibilité de les remplacer globalement par un pronom équivalent (ça/il/elle/le...) ou par un syntagme nominal (cette histoire + ce discours...), comme le montrent les remplacements entre parenthèses, dans les exemples (35-37): 
(35)

[comment se situe votre liberté ?] ce qui a été dit dans le préambule $(c ̧ a+? i l+c e$ discours) a été très fortement atténué dans les textes

(36)

on (le) transcrit ensuite ce qui a été dit (ça + ce discours) ça m'a rappelé euh mon enfance et ce qui avait été dit par mes parents (ça + ce discours)

(37)

je suis sûre qu'ils (l') entendent pas ce qui est raconté (ça + ce discours) une relative ou une complétive, il faudrait noter que, dans 14 cas de notre corpus, les VP au passif sont du côté de la matrice. Ils font office de verbes introducteurs de la parole et la forme suivante (que-P) correspond au contenu propositionnel exprimé. Cela apparait surtout dans les tours impersonnels, du type : il a été décidé/dit/demandé (que/ $s i / q u a n d+P)$. Ce rôle d'introducteur de parole se voit dans la possibilité de supprimer le VP utilisé pour ne garder que les propos rapportés. Ceci dit, une telle suppression entraine une prise en charge complète de ce qui est rapporté, comme le montrent les transformées (42a-44a) :

(42)

un jour je crois qu'ils ont demandé un exemple des cendriers dans les vestiaires de il a été répondu que les les femmes [oui] n'avaient pas besoin que normalement les femmes ne fumaient pas

(42a) les femmes n'avaient pas besoin normalement les femmes ne fumaient pas

(43)

il nous a été dit que c'était une formule transitoire

(43a) c'était une formule transitoire

(44)

et euh brusquement enfin d'une façon extrêmement maladroite il a été décidé de qu'on soit transféré dans l'autre école

(44a) on a été transféré dans l'autre école 


\subsubsection{Le passif périphrastique comme segment associé}

Outre son statut de dépendant microsyntaxique, régi par un verbe, un nom ou un adjectif, le passif périphrastique des VP, d'après notre corpus, peut aussi occuper des positions extraphrastiques (voir les fréquences de ces distributions dans le tableau A). Il entre, en fait, dans de nombreuses relations de dépendances macrosyntaxiques qu'il conviendrait de prendre en considération pour éviter d'avoir une description partielle de ce tour. Cette interdépendance macrosyntaxique se traduit globalement par un rattachement à un segment-noyau à l'aide de connecteurs syntaxiques (cf. une fois que c'est répondu c'est fini), ou sans (cf. bah il y a le l'arabe il est parléà Orléans).

Les distributions prosodiques observables peuvent assurer cette fonction (Morel \& Danon-Boileau, 1998). Ce sont ainsi ces liens transphrastiques diffus et polymodaux (cf. marques linguistiques, marquage prosodique, unicité du sujet abordé, isotopies lexicales, émotions et attitudes en jeu, tours de parole, etc.) qui permettent de définir les limites d'un paragraphe ou d'une période (Béguelin, 2002; Adam, 2005; RossiGensane, 2010). Le tableau A, ci-dessous, donne un aperçu de la fréquence des passifs périphrastiques relevés dans les ESLO concernant les VP au passif. Il en ressort que le passif n'est généralement pas un segment indépendant.

Tableau A. - Distribution des statuts micro et macrosyntaxiques des segments passifs des VP.

$\begin{array}{lrr}\text { Statut du passif périphrastique } & \text { Occ. } & \text { Fréq. \% } \\ \text { (Macro) Post-noyau (Macro) } & 79 & 30,98 \% \\ \text { (Micro) Complément de N } & 67 & 26,27 \\ \text { (Macro) Noyau } & 43 & 16,86 \\ \text { (Micro) complément de V } & 41 & 16,07 \\ \text { (Micro) ce qui P (= nominal) } & 20 & 7,84 \\ \text { (Macro) Pré-Noyau } & 19 & 7,45 \\ \text { (Macro) parenthèse } & 6 & 2,35\end{array}$

Comme noyau, on retrouve souvent des segments assertifs, où un contenu informationnel est partagé et qui peut aussi prendre la forme d'une réponse à une question. Parmi les autres réalisations possibles comme noyau, on peut avoir aussi des segments qui peuvent constituer, eux-mêmes, des questions, dont dépendent d'autres regroupements secondaires à gauche, à droite, ou sous forme de parenthétiques. Ainsi, dans l'exemple (45), le segment passif tout est expliqué constitue le noyau des regroupements environnants. On reconnait ce noyau, du point de vue syntaxique, à sa compatibilité, par exemple, avec les modalités négatives et interrogatives, conformément à ce qui est préconisé dans le cadre de l'école aixoise. Le prouve aussi la lexicalisation possible des pronoms dans ces exemples, aboutissant à des syntagmes à tête lexicale, comme en (45a-45c) et (46a-46c). Les contours prosodiques ponctuant les échanges cités confirment globalement ce constat. Ainsi, là où le noyau est affecté d'un accent conclusif ( \ \ou \\»), il y a toute une série de clauses délimitées par des accents continuatifs (notés «/ /), impliquant un maintien du tour de parole. On y voit une sorte de cadrage, assuré par une série de pré-noyaux (éléments préfixaux) et préparant le terrain à l'unité illocutoire. À droite du noyau, on peut aussi repérer un élément suffixal (un post-noyau) affecté d'un accent conclusif, copiant le noyau et constituant 
une sorte de greffe qui vient développer ce dernier. Cet élément sert généralement à justifier, détailler ou argumenter dans le même sens. Et, bien que cette dernière clause soit introduite par un lien syntaxique (parce que), elle reste en dehors de la rection du verbe expliquer au passif, ce que montre clairement la bizarrerie entrainée par son intégration dans un dispositif d'extraction en (45d) :

(45)

s'il y a un mot que je comprends pas/ ou un pays que je veux voir euh/ qu'est-ce qui se passe dans ce pays euh/ comme là/ tout est expliqué $\backslash$ parce que c'est le dernier Larousse sorti \\

(46)

[il vous arrive de les reprendre?]// euh/ rarement \ parce que on se surveille mutuellement pour ça $\backslash \backslash$ [oui $\backslash \backslash$ et dans les cas où vous les/ vous auriez à les reprendre ?//] amical $\backslash$ c'est dit amicalement $\backslash \backslash$

(45a) tout $n$ 'est pas expliqué

(46a) c'est pas dit amicalement

(45b) (est-ce que) tout est expliqué ?

(46b) (est-ce que) c'est dit amicalement?

(45c) le déroulement de l'activité est expliqué

(46c) le reproche est dit amicalement

(45d) ??c'est parce que c'est le dernier Larousse sorti que tout est expliqué

Dans les exemples (47-49), on a plutôt affaire à des post-noyaux. C'est le cas de 79 cas sur les 127 autres occurrences relevées. Ils viennent grosso modo fournir une précision ne faisant pas nécessairement partie de l'élément-noyau, tout en y apportant un important éclairage, de l'ordre d'une restriction, d'un rappel, d'une explication, ou d'une justification, etc. Notons que la connexion entre le noyau et son extension à droite peut se faire à l'aide d'un lien syntaxique ou sans (cf. puisque, parce que, car, étant donné que) et que le post-noyau en question n'est pas forcément régi par le verbe.

(47)

[est -ce que vous avez remarqué des justement dans votre travail euh/ où vous êtes en $\mathrm{p}$ - en contact euh/ un peu un spectre large de la population quoi $\backslash$ d'autres langues parlées à Orléans \est -ce qu'il y a d'autres langues ?//] on a le droit// [bah bah oui oui oui $\backslash \backslash$ bah il y a le l'arabe $\backslash$ il est parlé à Orléans $\backslash \backslash$

(48)

la plupart du travail ne demande pas une connaissance spéciale \bon\[oui d'accord] puisque tout étant détaillé sur le/ [oui oui oui oui oui oui] tout étant expliqué sur le/ [oui] sur l'ouvrage \\}

(49)

c'était une une arrière grand-mère ou un arrière grand-père/ qui était hémiplégique/ d'ailleurs qui qui a fini par se brûler dans le feu \enfin qui a eu des tas d'aventures \ça ça m'a été raconté par euh ma grand-mère \qui était [oui] qui était totalement illettrée $\backslash \backslash$ [ça a dû être une forte femme hein//] ah c'était une maitresse femme $\backslash \backslash[$ oui $\backslash \backslash]$

31 En ce qui concerne les emplois du passif comme élément préfixal (pré-noyau), on a souvent une sorte de cadrage précédant et préparant le terrain à l'unité illocutoire (constituée par le noyau). On en a relevé 19 cas. Ce type de segments peut être marqué prosodiquement par un accent continuatif à la fin (50-51), ce qui prend parfois dans les constructions macrosyntaxiques la forme d'un regroupement liminaire construit à l'aide d'un subordonnant, tel que si, vu que, comme, etc., et qui a un rôle plutôt extraprédicatif globalement, comme le montre l'incompatibilité de son intégration dans un dispositif d'extraction, en (50a-51a). Ainsi, bien que le segment passif dans son 
emploi préfixal soit rattaché sur le plan sémantique et pragmatique au noyau, on voit clairement qu'il n'est pas sous sa rection syntaxique :

(50)

une fois que c'est répondu/ c'est fini\

(51)

comme ça a été dit/ notre laboratoire est plutôt connu pour l'histoire ancienne et la numismatique \un peu moins pour euh d'autres recherches $\backslash$

(50a) ??c'est une fois que c'est répondu que c'est fini

(51a) ??c'est comme ça a été dit que notre laboratoire est plutôt connu pour l'histoire ancienne et la numismatique

Nous avons relevé aussi 6 occurrences où le passif est analysable comme une parenthèse. On reconnait ces parenthèses à la fois à leur caractère facultatif du point de vue syntaxique, puisque leur suppression reste possible, comme dans (52-54), sans trop affecter le sens et la construction globalement. Et, du point de vue pragmatique, il y a souvent un rappel de ce qui a déjà été dit ou décidé ailleurs par une certaine autorité, ce qui peut raffermir l'idée défendue (cf. il y a un précédent à ce que l'on dit) ou constituer, dans certains autres cas, une sorte de dégagement de la responsabilité de ce que l'on rapporte. Enfin, du point de vue suprasegmental, on a très souvent une intonation plutôt plate et des contours sans intensité (-) :

(52)

je veux pas le croire hein mais c'est une question - et c'est ça a été affirmé par certains - est-ce que c'est pas machiavélique d'avoir des maisons de la culture quand on est un gouvernement qui est contre la culture finalement

(53)

euh moi je crois qu'on est très influencé et le problème actuellement euh - c'est un problème qui a été évoqué y a pas longtemps dans le Monde voyez l'article qui est là [ah oui] Orléans à la recherche d'une vocation hein euh [...] - le problème de savoir si on ne devrait pas redistribuer les régions et réduire le nombre des régions...

(54)

je pense que c- et même ouvrir pendant midi - ça a été demandé souvent par la mairie d'Orléans - que surtout le vendredi...

Notons que contrairement aux incises impliquant des verbes introducteurs de la parole que l'on rencontre surtout à l'écrit, ces éléments parenthétiques au passif n'introduisent aucune parole précise (ce ne sont pas des cas de discours rapporté), mais rappellent, dans les exemples relevés, le caractère commun ou consensuel de ce qui est dit (une sorte d'écho), ce qui relève de toute évidence d'une stratégie de persuasion largement utilisée dans les échanges spontanés.

\section{Bilan et conclusion}

L'examen des données orales a permis de se rendre compte de la diversité des découpages possibles à associer aux différentes réalisations d'un tour grammatical comme le passif périphrastique en français parlé. La non-coextensivité du passif à l'étendue de la phrase est désormais un fait indéniable. Un tel fait n'aurait pas pu être établi avec les données utilisées habituellement relevant le plus souvent de phrases forgées ou de phrases sorties de leurs contextes. Le recours à l'oral a permis aussi de réhabiliter certaines catégories marginalisées, car non prises en charge justement par la syntaxe de la phrase. C'est le cas des segments macrosyntaxiques des passifs des VP. Cela concerne près de la moitié des emplois relevés. Ce recours a permis aussi de mettre 
en lumière les emplois non autonomes (voir tableau A), avec 50,19\% des cas. Le passif y est un simple segment hypotaxique - formes souvent marginalisées dans les travaux connus. De fait, dans ce tournant historique où l'oralité et les corpus oraux commencent à occuper une place importante dans les pratiques et les investigations linguistiques, il devient indispensable de reconsidérer les gloses et analyses classiques circulant sur le passif à la lumière de ce qu'apporte l'oralité. Une confrontation aux données orales pourra amener à ratifier les descriptions pertinentes répertoriées, à compléter le panorama global de la caractérisation de ce tour et, le cas échéant, à revisiter les gloses et les hypothèses qui semblent conditionnées par la nature des données utilisées.

\section{BIBLIOGRAPHIE}

ADAM, Jean-Michel. (2005). La linguistique textuelle. Introduction à l'analyse textuelle des discours. Armand Colin.

ANSCOMBRE, Jean-Claude. (2015). Verbes d'activité de parole, verbes de parole et verbes de dire : des catégories linguistiques? Langue française, 186, 103-121.

AvANZI, Mathieu. (2007). Regards croisés sur la notion de macro-syntaxe. Travaux neuchâtelois de linguistique, 47, 39-58.

BÉGUELIN, Marie-José. (2002). Clause, période ou autre ? La phrase graphique et la question des niveaux d'analyse. Verbum, XXIV(1-2), 85-108.

BEnzitoun, Christophe. (2010). Quelle(s) unité(s) syntaxique(s) maximale(s) en français parlé ? Discussions autour de quelques problèmes rencontrés. Travaux de linguistique, 60, 109-126.

BERRENDONNER, Alain. (1990). Pour une macro-syntaxe. Travaux de linguistique, 21, 25-36.

BERRENDONNER, Alain. (2003). Éléments pour une macro-syntaxe : actions communicatives, types de clauses, structures périodiques. Dans A. Scarano (dir.), Macro-syntaxe et pragmatique. L'analyse linguistique de l'oral (p. 93-110). Bulzoni Editore.

BERRENDONNER, Alain. (2011). Unités syntaxiques et unités prosodiques. Langue française, 170, 81-94.

BLANCHE-BENVENISTE, Claire. (2010). Approches de la langue parlée en français. Ophrys.

Blanche-Benveniste, Claire, Deulofeu, José, Stefanini, Jean \& Van den Eynde, Karel. (1984). Pronom et syntaxe : l'approche pronominale et son application au français. SELAF.

BlanCHE-BENVENISTE, Claire \& JEANJEAN, Colette. (1987). Le français parlé : transcription et édition. INALF.

BLOOMFIELD, Leonard. (1970-1933). Le langage. Payot.

BRAHIM, Ahmed. (1996). L'occultif: hypothèse pour un traitement trans-linguistique du " passif» et des structures apparentées. Association tunisienne de linguistique.

DIK, Simon Cornelis. (1989). The Theory of Functional Grammar. Foris. 
DuBoIs, Jean. (1967). Grammaire structurale du français. Le verbe. Larousse.

ELDUAYEN, Luis Gastón. (2007). Introduire le discours d'autrui : actes de parole et verbes «secondaires » dans la presse franco-espagnole. RESLA, 20, 37-58.

GAATONE, David. (1998). Le passif en français. Duculot.

GROss, Maurice. (1975). Méthodes en syntaxe. Hermann.

Gross, Maurice. (1981). Les bases empiriques de la notion de prédicat sémantique. Langages, 63, 7-52.

GROUPE DE FRIBOURG. (2012). Grammaire de la période. Peter Lang.

HAMMA, Badreddine. (2015). Agent passif en par et sujet actif : les dessous d'un contraste. RSP, 37, 61-83.

HAMmA, Badreddine. (2017). Tentative de classification des « compléments d'agent » dans les phrases passives achevées et dans les énoncés longs à sens passif. ELA, 187(3), 311-324.

HAMMA, Badreddine. (2020a). Les figures de l'agent et du complément d'agent dans l'usage et dans le métalangage. Dans M. Meulleman, S. Palma \& A. Theissen (dir.), Liber Amicorum. Mélanges offerts à Emilia Hilgert (p. 147-272). Éditions et Presses universitaires de Reims (Épure).

HAMMA, Badreddine. (2020b). Variations diamésiques autour du passif périphrastique en français. Dans M. Saiz-Sánchez, A. Rodríguez Somolinos \& S. Gómez-Jordana Ferary (dir.), Marques d'oralité et représentation de l'oral en français (p. 43-66). Presses universitaires Savoie Mont Blanc.

HAMMA, Badreddine, TARDIF, Amélie \& BADIN, Flora. (2017). Le passif à l'oral. Fiche FRACOV : $<$ www.ortolang.fr/market/corpora/fracov?path=\%2F>.

HASELOW, Alexander. (2016). Micro- et macro-syntaxe : sur deux principes de catégorisation cognitive en langue orale spontanée. Modèles linguistiques, 74. <https://doi.org/10.4000/ml.1895>. LAMIRoy, Béatrice \& CHAROLLES, Michel. (2008). Les verbes de parole et la question de l'(in)transitivité. Discours, 2. <https://doi.org/10.4000/discours.3232>.

LE GoffIC, Pierre. (1993). Grammaire de la phrase française. Hachette.

LE GoffIC, Pierre. (2016). « Y a-t-il une syntaxe au-delà de la phrase? » Retour sur une question lancinante. Dans L. Sarda, D. Vigier \& B. Combettes (dir.), Connexion et indexation : ces liens qui tissent le texte. ENS Éditions. <https://doi.org/10.4000/books.enseditions.6866>.

MATHIEU-COLAS, Michel. (2006). Les classes de verbes : syntaxe et sémantique. Dans J. Bacha \& S. Mejri (dir.), Le traitement du lexique. Catégorisation et actualisation (p. 10-24).

MEL'čUK, Igor. (2004). Actants in Semantics and Syntax. Linguistics, 42(1), 1-66.

Morel, Marie-Annick \& DANON-BOILEAU, Laurent. (1998). Grammaire de l'intonation. Ophrys. RieGEL, Martin, Pellat, Jean-Christophe \& Rioul, René. (1994-2009). Grammaire méthodique du français. Presses universitaires de France.

RoSSI-GENSANE, Nathalie. (2010). Encore quelques remarques sur la phrase. La linguistique, 46(2), 69-107.

SABIO, Frédéric. (2006). Phrases et constructions verbales : quelques remarques sur les unités syntaxiques dans le français parlé. Dans D. Lebaud, C. Paulin \& K. Ploog (dir.), Constructions verbales et production de sens (p. 127-139). Presses universitaires de Franche-Comté. 


\section{NOTES}

1. Le passif périphrastique, la forme canonique du passif en français, se définit comme une disposition actancielle construite nécessairement (1) autour de la périphrase "être + $\mathrm{V}_{\text {PPé }}$ "; (2) avec un sujet (No) renvoyant à l'argument interne du verbe (coïncidant souvent avec le patient). Il est à noter que pour diverses raisons, certains arguments peuvent être absents en surface tout en restant accessible (voir infra § 3).

2. ESLO est un corpus en libre accès sur <http://eslo.huma-num.fr> ou <https://cocoon.humanum.fr>.

3. TXM est un logiciel de textométrie open-source conçu et développé par Heiden, Magué et Pincemin en 2010. Il permet une extraction étiquetée des données linguistiques. Ici, nous utilisons la version 0.8.1. Se rendre à l'adresse <http://textometrie.ens-lyon.fr/> pour plus de détails sur le logiciel et ses fonctionnalités.

4. Certains verbes n'ont donné aucun résultat: dénigrer, énoncer, relater, etc., ou aussi rétorquer, répliquer, etc. (ici à l'impersonnel), ce qui pourrait s'expliquer par une question de genre de discours.

5. Dans les exemples cités des ESLO, par gain de place, on ne reprend à chaque fois que ce qui est pertinent pour l'analyse. L'accès à un contexte plus large reste possible sur la plateforme ESLO. Ici, nous utilisons l'italique pour souligner les segments ciblés pour chaque point de l'analyse de nos VP au passif et les crochets renvoient à l'interaction concomitante de l'interlocuteur. Pour la notation des contours prosodiques des exemples, nous utilisons le symbole «/» pour un accent continuatif, «// " pour un contour conclusif avec une intonation montante, « \» pour noter un contour conclusif avec une continuation immédiate, « \\» pour un contour conclusif suivi d'une pause et «-- " pour les intonations plates.

6. Il s'agit d'une longue digression parenthétique; ici, nous n'en reprenons que la partie renfermant un passif.

\section{RÉSUMÉS}

L'objectif de cette étude est de faire le point sur les apports de l'oralité et des corpus oraux à l'étude du passif. Au cœur de ces apports, il conviendrait, selon nous, de citer la question du statut grammatical global de ce tour et de son étendue dans un discours non contrôlé, en termes de coextensivité (le passif dans l'usage ne coïncidant pas toujours avec l'étendue phrastique). Il en ressort que l'espace de la phrase ne permet pas de rendre compte de toutes les constructions passives observables dans le discours et marginalise, par exemple, ses emplois macrosyntaxiques, ce qui pourrait avoir une incidence sur les gloses et les analyses connues sur ce tour. Notre démonstration concernera ici les verbes de parole dans leur emploi au passif périphrastique à l'oral.

The aim of this study is to take stock of the contributions of orality and oral corpora to the study of passive in French. At the heart of these contributions, it would be appropriate, in our opinion, to mention the question of the global grammatical status of this turn and its extent in non-controlled discourse, in terms of coextensiveness (passive uses do not always coincide with the limits of the sentence). It emerges that the narrow frame of the sentence does not allow accounting for all the passive uses that can be observed in the discourse and leaves aside, for 
instance, macrosyntactic ones, which could have an impact on the known glosses and analyses of the passive construction. Our demonstration will concern speech verbs in their periphrastic passive uses in the oral speech.

INDEX

Mots-clés : passif, passif périphrastique, microsyntaxe, macrosyntaxe, oral, verbes de parole Keywords : passive voice, periphrastic passive, microsyntax, macrosyntax, oral speech, speech verbs

\section{AUTEUR}

\section{BADREDDINE HAMMA}

LLL, UMR 7270, Université d'Orléans badreddine.hamma@univ-orleans.fr 